\title{
Bifurcation Analysis of a Time- and Space-Discrete Predator-Prey Model with Holling II Function Response
}

\author{
Tianjiao Zhang ${ }^{1}$, Yan Meng ${ }^{2, *}$ and Yongan $\mathrm{Ye}^{3}$ \\ ${ }^{1}$ School of Mathematics and Physics University of Science and Technology, Beijing \\ ${ }^{2}$ School of Mathematics and Physics University of Science and Technology, Beijing \\ ${ }^{3}$ Dongzhimen Hospital Beijing University of Chinese Medicine \\ ${ }^{*}$ Corresponding author
}

\begin{abstract}
In this paper, a space- and time-discrete predatorprey model with Holling type II function response is investigated. The mortality of predator is variable, which is related to the population density. The model is given by a coupled map lattice framework, it takes a nonlinear relationship between predatorprey reaction stage and dispersal stage. The stability of equilibrium point and the parameter conditions for the Hopf bifurcation are obtained when the diffusion is absent. After adding the diffusion, we obtained the parameter conditions for the Turing instability. Numerical simulations verify the theoretical analysis and show a series of spatial patterns with the change of the parameters.
\end{abstract}

Keywords-discrete model; coupled map lattice; hopf bifurcation; turing instability; pattern formation

\section{INTRODUCTION}

The predation relationship of different species is a kind of basic ecological relationship widely existing in nature. Since Lotka and Volterra have put forward the basic model, it has attract more and more researchers' attention[1-2]. With the development of the predator-prey model, the dynamics behaviors are becoming more and more abundant and complex, such as bifurcations, Turing instability, chaos and some other phenomenon[3-5].

Turing found that diffusion could destabilize the stable equilibrium. Such an instability is called Turing instability or diffusion-driven instability. These theories were confirmed by some chemical experiments[6,7].

Most of the researchers studied time- and space-continuous predator-prey model. However, some practical problems suitable for studying with the discrete model, such as scattered habitats, biological population of non-overlapping. It is found that the discrete model is more accurate than the corresponding continuous model when we describe the population dynamics[8]. Many studies have shown that the application of discrete dynamics model can obtain better results in the study of predator-prey system[9].

The outline of this paper is as follows. In Section 2, we give the time- and space-discrete model. In Section 3, we discuss that the stability of equilibrium points and the parameter conditions of the bifurcation when the diffusion is absent. Furthermore, we obtain the parameter conditions for the Turing instability after adding the diffusion term. In Section 4, details of numerical simulation are given to verify the theoretical results. Concluding remarks are given in Section 5.

\section{The TIME- AND SPACE-Discrete PREDATOR-PREY MODEL}

Cavani and Farkasin consider the following continuous predator-prey model with diffusion[10]

$$
\left\{\begin{array}{l}
\frac{\partial N(x, t)}{\partial t}=N \varepsilon\left(1-\frac{N}{K}\right)-\frac{a N P}{\beta+N}+d_{1} \nabla^{2} N, x \in \Omega, t>0 \\
\frac{\partial P(x, t)}{\partial t}=\frac{b N P}{\beta+N}-\frac{P(\gamma+\delta P)}{1+P}+d_{2} \nabla^{2} P, x \in \Omega, t>0 \\
N_{x}(0, t)=P_{x}(0, t)=0, N_{x}(l \pi, t)=P_{x}(l \pi, t)=0, t>0 \\
N_{x}(x, 0)=N_{0}(x) \geq 0, P_{x}(x, 0)=P_{0}(x) \geq 0, x \in \bar{\Omega}
\end{array}\right.
$$

where $N$ and $P$ represent the population density of prey and predator, respectively. $t$ denotes time; $a$ is the energy conversion rate with respect to prey; $b$ represents the ratio of energy to predator population during predation; $\varepsilon$ is maximum per growth rate of the prey; $K$ and $\beta$ are the environmental carrying capacity and conversion rate with respect to the prey; $\gamma$ and $\delta$ are the minimal mortality and the limiting mortality of the predator (the natural assumption is $\gamma<\delta$ ). $d_{1}$ and $d_{2}$ are diffusion coefficients.

The condition $\gamma<\frac{\beta K}{\beta+K}$ is needed to ensure that the system has a positive equilibrium point[10]. And according to the Eqs.(1), we can calculate the following equation set the figure of Eqs.(2) shows that the system must have positive equilibrium point(see FIGURE I ).

$$
\left\{\begin{array}{l}
P_{1}(N)=\frac{\varepsilon(K-N)(\beta+N)}{a K} \\
P_{2}(N)=\frac{(b-\gamma) N-\beta \gamma}{(\delta-b) N+\beta \delta}
\end{array}\right.
$$




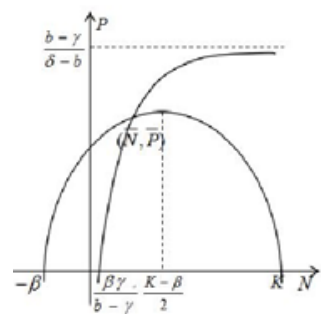

FIGURE I. THE EXISTENCE OF POSITIVE EQUILIBRIUM POINT.

The continuous model in Eqs.(1) is discretized in a coupled map lattice frame-work, we set a time interval of $\tau$ and a space interval $h$, and a two-dimensional rectangular area divided into $n \times n$ squares (note that the sides of each grid are $h$ ). Each grid represents a spot, and each grid has two variables, the density of prey population and predator population. Under the influence of system dynamics, the density of predator and prey in each grid can change with time because of the local external and internal relations, and the migration and diffusion between the grids. On this basis, we define two state variables $N_{(i, j, m)}$ and $P_{(i, j, m)}\left(i, j \in\{1,2, \ldots, n\}, m \in Z^{+}\right)$, which represent the prey density and the predator density in $(i, j)$ site and at $m$ th iteration (with initial time $t_{0}$, the time at $m$ th iteration is $\left.t_{0}+m \tau\right)$.

In the coupled map lattice framework, the dynamics at each step iteration of the model consists of a reaction stage and a dispersal stage. The reaction stage is described by

$$
\begin{aligned}
& N_{(i, j, m+1)}=N_{(i, j, m)}+\tau\left(\varepsilon N_{(i, j, m)}\left(1-\frac{N_{(i, j, m)}}{K}\right)-\frac{a N_{(i, j, m)} P_{(i, j, m)}}{\beta+N_{(i, j, m)}}\right) \\
& P_{(i, j, m+1)}=P_{(i, j, m)}+\tau\left(\frac{b N_{(i, j, m)} P_{(i, j, m)}}{\beta+N_{(i, j, m)}}-P_{(i, j, m)} \frac{r+\delta P_{(i, j, m)}}{1+P_{(i, j, m)}}\right)
\end{aligned}
$$

the dispersal stage is obtained by discretizing the spatial term of Eqs.(1),

$$
\begin{aligned}
& N_{(i, j, m)}^{\prime}=N_{(i, j, m)}+\frac{\tau}{h^{2}} d_{1} \nabla_{d}^{2} N_{(i, j, m)} \\
& P_{(i, j, m)}^{\prime}=P_{(i, j, m)}+\frac{\tau}{h^{2}} d_{2} \nabla_{d}^{2} P_{(i, j, m)}
\end{aligned}
$$

$\nabla_{d}^{2}$ is the discrete form of Laplacian operator, and shown as the following

$$
\begin{aligned}
& \nabla_{d}^{2} N_{(i, j, m)}=N_{(i+1, j, m)}+N_{(i-1, j, m)}+N_{(i, j+1, m)}+N_{(i, j-1, m)}-4 N_{(i, j, m)} \\
& \nabla_{d}^{2} P_{(i, j, m)}=P_{(i+1, j, m)}+P_{(i-1, j, m)}+P_{(i, j+1, m)}+P_{(i, j-1, m)}-4 P_{(i, j, m)}
\end{aligned}
$$

where dispersal is limited to the nearest neighbors.

In discrete model, we set periodic boundary conditions as the following,

$$
\begin{aligned}
& N_{(i, 0, m)}=N_{(i, n, m)}, N_{(i, 1, m)}=N_{(i, n+1, m)}, N_{(0, j, m)}=N_{(n, j, m)}, \\
& N_{(1, j, m)}=N_{(n+1, j, m)}, P_{(i, 0, m)}=P_{(i, n, m)}, P_{(i, 1, m)}=P_{(i, n+1, m)}, \\
& P_{(0, j, m)}=P_{(n, j, m)}, P_{(1, j, m)}=P_{(n+1, j, m)}
\end{aligned}
$$

The combination of Eqs.(3-5) describes the space- and timediscrete predator-prey model. All the parameters used in the discrete model are positive and the values of $N_{(i, j, m)}$ and $P_{(i, j, m)}$ are non-negative.

Next, we use the bifurcation theory of discrete model to study the Hopf bifurcation and the Turing instability of the discrete predator model.

\section{CONDITIONS OF THE EXISTENCE OF HOPF BIFURCATION AND TURING INSTABILITY}

\section{A. The Stability of the Equilibrium Point}

The homogeneous stationary states of the discrete model requires $\nabla_{d}^{2} N_{(i, j, m)}=0$ and $\nabla_{d}^{2} P_{(i, j, m)}=0 \quad\left(i, j \in\{1,2,3, \ldots, n\}, m \in Z^{+}\right)$. That is, we just need to discuss Eqs.(3) .the equilibrium point of the Eqs.(4) is calculated as

$$
\begin{array}{ll}
\left(N_{0}, P_{0}\right)=(0,0) & \left(N_{1}, P_{1}\right)=(K, 0) \\
\left(N_{2}, P_{2}\right)=\left(0,-\frac{r}{\delta}\right) & \left(N_{3}, P_{3}\right)=(\bar{N}, \bar{P})
\end{array}
$$

It is easy to show that the system must be have positive equilibrium point $\left(N_{3}, P_{3}\right)$. The expression of it is too complex, we use $(\bar{N}, \bar{P})$ to represent it.

Applying the Jacobian matrix of Eqs.(7),

$$
J(N, P)=\left(\begin{array}{cc}
1+\tau\left(\varepsilon\left(1-\frac{2 N}{K}\right)-\frac{a \beta P}{(\beta+N)^{2}}\right) & -\frac{\tau a N}{\beta+N} \\
\frac{\tau \beta b P}{(\beta+N)^{2}} & 1+\tau\left(\frac{b N}{\beta+N}-\delta-\frac{r-\delta}{(1+v)^{2}}\right)
\end{array}\right)
$$

substituting the equilibrium points of (7) into (8), we calculate two characteristic values of the corresponding Jacobian matrix, $\lambda_{1}$ and $\lambda_{2}$. According the following criteria[11] : (1) if $\left|\lambda_{1}\right|<1$ and $\left|\lambda_{2}\right|<1$, then the corresponding fixed point is a stable node or a stable focus; (2) if $\left|\lambda_{1}\right|>1$ and $\left|\lambda_{2}\right|>1$, then the corresponding fixed point is an unstable node or an unstable focus; (3) if $\left|\lambda_{1}\right|>1$ and $\left|\lambda_{2}\right|<1$ or $\left|\lambda_{1}\right|<1$ and $\left|\lambda_{2}\right|>1$, then the corresponding fixed point is a saddle point, which is also unstable.

From the above criteria, it is easy to conclude that $\left(N_{0}, P_{0}\right)$ and $\left(N_{1}, P_{1}\right)$ are unstable. When $\frac{b K}{\beta+K}<\gamma<\frac{2}{\tau}+\frac{b K}{\beta+K}$ and $0<\tau \varepsilon<2$, $\left(N_{2}, P_{2}\right)$ is stable. And the system is stable at $\left(N_{3}, P_{3}\right)$, each parameter should satisfies the following conditions: 


$$
\begin{aligned}
& a_{11}+a_{22}<1+a_{11} a_{22}-a_{12} a_{21} \\
& a_{11}+a_{22}>-\left(1+a_{11} a_{22}-a_{12} a_{21}\right) \\
& a_{11} a_{22}-a_{12} a_{21}<1 .
\end{aligned}
$$

where

$$
\begin{array}{ll}
a_{11}=1+\tau \varepsilon\left(1-\frac{2 N_{3}}{K}\right)-a_{21} & a_{12}=-\tau\left(\delta+\frac{r-\delta}{1+v}\right) \\
a_{21}=\frac{\tau \beta \varepsilon^{2}}{a v}\left(1-\frac{N_{3}}{K}\right) & a_{22}=1-\frac{\tau \delta P_{3}}{1+P_{3}}+\frac{a_{21} P_{3}}{1+P_{3}}
\end{array}
$$

Next, we discuss the equilibrium point $\left(N_{3}, P_{3}\right)$.

\section{B. Conditions for Hopf Bifurcation}

According to the discrete system bifurcation theory[12], the Hopf bifurcation is independent in space, so next we will not consider the diffusion term in the study of Hopf bifurcation, i.e., $\nabla_{d}^{2} N_{(i, j, m)}=0$ and $\nabla_{d}^{2} P_{(i, j, m)}=0$. We choose the time interval $\tau$ as the bifurcation parameters of Hopf bifurcation, when the other parameters fixed, we study the parameters $\tau$ for the stability of the role and influence of the system.

The two eigenvalues of the Jacobi matrix of $\left(N_{3}, P_{3}\right)$ are as follows

$$
\lambda_{1,2}=\frac{1}{2}\left(a_{11}+a_{22} \pm \sqrt{\left(a_{11}-a_{22}\right)^{2}+4 a_{12} a_{21}}\right)
$$

The first condition for the appearance of Hopf bifurcation $\lambda_{1}$ and $\lambda_{2}$ is a pair of complex conjugate with modulus one, i.e.,

$$
\left(a_{11}-a_{22}\right)^{2}+4 a_{12} a_{21}<0
$$

when $a_{11} a_{22}-a_{12} a_{21}=1$, i.e.,

$$
\tau=\tau_{0}=\frac{\frac{\beta \varepsilon^{2}}{a P_{3}}\left(1-\frac{N_{3}}{K}\right)^{2}-\frac{P_{3}(r-\delta)}{\left(1+P_{3}\right)^{2}}-\varepsilon\left(1-\frac{2 N_{3}}{K}\right)}{\frac{\varepsilon P_{3}(r-\delta)}{\left(1+P_{3}\right)^{2}}\left(\left(1-\frac{2 N_{3}}{K}\right)-\frac{\beta \varepsilon^{2}}{a P_{3}}\left(1-\frac{N_{3}}{K}\right)^{2}\right)-\frac{\beta \varepsilon^{2}}{a P_{3}}\left(1-\frac{N_{3}}{K}\right)^{2}\left(\delta-\frac{r-\delta}{1+P_{3}}\right)} \neq 0
$$

we have $\left|\lambda_{1}\right|=\left|\lambda_{2}\right|=1$.

Next, we change the $\left(N_{3}, P_{3}\right)$ to the origin by the following transformation

$$
\begin{aligned}
& u=N-N_{3} \\
& v=P-P_{3}
\end{aligned}
$$

$$
\left(\begin{array}{l}
u \\
v
\end{array}\right) \rightarrow\left(\begin{array}{l}
\left.b_{11} u+b_{12} v+\frac{b_{13}}{2} u^{2}+b_{14} u v+\frac{b_{15}}{2} v^{2}+\frac{b_{16}}{6} u^{3}+\frac{b_{17}}{2} u^{2} v+\frac{b_{18}}{2} u v^{2}+\frac{b_{19}}{6} v^{3}+O(|| u|+| v \mid)^{4}\right) \\
\left.b_{21} u+b_{22} v+\frac{b_{23}}{2} u^{2}+b_{24} u v+\frac{b_{25}}{2} v^{2}+\frac{b_{26}}{6} u^{3}+\frac{b_{27}}{2} u^{2} v+\frac{b_{28}}{2} u v^{2}+\frac{b_{29}}{6} v^{3}+O(|u|+|v|)^{4}\right)
\end{array}\right)
$$

where $O\left((|u|+|v|)^{4}\right)$ describes a function with order at least four in the variables $(u, v)$, and

$$
\begin{array}{lllll}
b_{11}=a_{11} & b_{12}=-\frac{a}{b} a_{12} \quad b_{13}=-\frac{2 \tau \varepsilon}{K}+\frac{2 \tau \beta a N_{3}}{\left(\beta+N_{3}\right)^{2}} & b_{14}=-\frac{\tau \beta a}{\left(\beta+N_{3}\right)^{2}} \\
b_{15}=0 & b_{16}=-\frac{6 \tau \beta a N_{3}}{\left(\beta+N_{3}\right)^{4}} & b_{17}=\frac{2 \tau \beta a}{\left(\beta+N_{3}\right)^{2}} & b_{18}=0 \\
b_{19}=0 & b_{21}=-\frac{a}{b} a_{21} \quad b_{22}=a_{22} & b_{23}=-\frac{2 \tau \beta b P_{3}}{\left(\beta+N_{3}\right)^{3}} \\
b_{24}=\frac{\tau \beta b}{\left(\beta+N_{3}\right)^{2}} & b_{25}=\frac{2 \tau \beta(r-\delta)}{\left(1+P_{3}\right)^{3}} \quad b_{26}=\frac{6 \tau b N_{3} P_{3}}{\left(\beta+N_{3}\right)^{4}} & b_{27}=-\frac{2 \tau \beta b}{\left(\beta+N_{3}\right)^{3}} \\
b_{28}=0 & b_{29}=-\frac{6 \tau(r-\delta)}{\left(1+P_{3}\right)^{4}} & &
\end{array}
$$

Under the conditions (11) and (12), the eigenvalues of the Jacobi matrix of the map(15) at equilibrium point are still conjugate complex numbers with modulus one. Similar to the previous method of discussion, we can write the corresponding characteristic equation and calculate two eigenvalues. In order to facilitate subsequent discussions, the two eigenvalue values can be written in the following form

$$
\lambda\left(\tau_{0}\right), \bar{\lambda}\left(\tau_{0}\right)=\frac{-p\left(\tau_{0}\right)}{2} \pm \frac{i}{2} \sqrt{4 q\left(\tau_{0}\right)-p^{2}\left(\tau_{0}\right)}=\alpha_{1} \pm i \alpha_{2}
$$

where

$$
\begin{aligned}
p\left(\tau_{0}\right)= & -2-\tau \varepsilon\left(1-\frac{2 N_{3}}{K}\right)+\frac{\tau \beta \varepsilon^{2}}{a P_{3}}\left(1-\frac{N_{3}}{K}\right)^{2}-\frac{\tau P_{3}(r-\delta)}{\left(1+P_{3}\right)^{2}} \\
q\left(\tau_{0}\right)= & 1+\tau_{0}\left(\frac{P_{3}(r-\delta)}{\left(1+P_{3}\right)^{2}}+\varepsilon\left(1-\frac{2 N_{3}}{K}\right)-\frac{\beta \varepsilon^{2}}{a P_{3}}\left(1-\frac{N_{3}}{K}\right)^{2}\right) \\
& +\tau_{0}^{2}\left(\frac{\varepsilon P_{3}(r-\delta)}{\left(1+P_{3}\right)^{2}}\left(1-\frac{2 N_{3}}{K}\right)-\frac{\beta \varepsilon^{2} P_{3}(r-\delta)\left(2 P_{3}+1\right)}{a P\left(1+P_{3}\right)^{2}}\left(1-\frac{N_{3}}{K}\right)^{2}\right)
\end{aligned}
$$

since the modulus of eigenvalues is one, there is $|\lambda|=\sqrt{q\left(\tau_{0}\right)}=1$. Another condition of the existence of Hopf bifurcation is

$$
d=\left.\frac{d|\lambda(\tau)|}{d \tau}\right|_{\tau=\tau_{0}}=-\frac{1}{2}\left(\frac{P_{3}(r-\delta)}{\left(1+P_{3}\right)^{2}}+\frac{\varepsilon N_{3}}{K}+\frac{\varepsilon N_{3}}{a P_{3}}\left(1-\frac{N_{3}}{K}\right)^{2}\right) \neq 0
$$

In addition, the following conditions are also required

$$
\left(\lambda\left(\tau_{0}\right)\right)^{\theta} \neq 1, \theta \neq 1,2,3,4
$$

which is equivalent to

$$
p\left(\tau_{0}\right) \neq-2,0,1,2
$$



is

It is known by Eqs.(14), $p\left(\tau_{0}\right) \neq \pm 2$. The requirement of $p\left(\tau_{0}\right) \neq 0,1$

$$
\tau_{0} \varepsilon\left(1-\frac{2 N_{3}}{K}\right)-\frac{\tau \beta \varepsilon^{2}}{a P_{3}}\left(1-\frac{N_{3}}{K}\right)^{2}+\frac{\tau P_{3}(r-\delta)}{\left(1+P_{3}\right)^{2}} \neq-3,-2
$$

Next the normal form of (13) when $\tau=\tau_{0}$ is studied. Applying the transformation

$$
\left(\begin{array}{l}
u \\
v
\end{array}\right)=\left(\begin{array}{cc}
b_{12} & 0 \\
\alpha_{1}-b_{11} & -\alpha_{2}
\end{array}\right)\left(\begin{array}{l}
U \\
V
\end{array}\right)
$$

then the map (14) becomes

$$
\left(\begin{array}{l}
U \\
V
\end{array}\right) \rightarrow\left(\begin{array}{cc}
\alpha_{1} & -\alpha_{2} \\
\alpha_{2} & \alpha_{1}
\end{array}\right)\left(\begin{array}{l}
U \\
V
\end{array}\right)+\frac{1}{b_{11} \alpha_{2}}\left(\begin{array}{l}
F_{1}(U, V) \\
G_{1}(U, V)
\end{array}\right)
$$

where

$$
\begin{aligned}
F_{1}(U, V)= & \frac{b_{13} \alpha_{2}}{2} u^{2}+b_{14} \alpha_{2} u v+\frac{b_{15} \alpha_{2}}{2} v^{2}+\frac{b_{16} \alpha_{2}}{6} u^{3}+\frac{b_{17} \alpha_{2}}{2} u^{2} v \\
& +\frac{b_{18} \alpha_{2}}{2} u v^{2}+\frac{b_{19} \alpha_{2}}{6} v^{3}+\left(O(|U|+|V|)^{4}\right) \\
G_{1}(U, V)= & \left(\frac{b_{13}\left(\alpha_{1}-b_{11}\right)}{2}-\frac{b_{12} b_{23}}{2}\right) u^{2}+\left(b_{14}\left(\alpha_{1}-b_{11}\right)-b_{12} b_{24}\right) u v \\
& +\left(\frac{b_{15}\left(\alpha_{1}-b_{11}\right)}{2}-\frac{b_{12} b_{25}}{2}\right) v^{2}+\left(\frac{b_{16}\left(\alpha_{1}-b_{11}\right)}{6}-\frac{b_{12} b_{26}}{6}\right) u^{3} \\
& +\left(\frac{b_{17}\left(\alpha_{1}-b_{11}\right)}{2}-\frac{b_{12} b_{27}}{2}\right) u^{2} v+\left(\frac{b_{18}\left(\alpha_{1}-b_{11}\right)}{2}-\frac{b_{12} b_{28}}{2}\right) u v^{2} \\
& +\left(\frac{b_{19}\left(\alpha_{1}-b_{11}\right)}{2}-\frac{b_{12} b_{29}}{2}\right) v^{3}+\left(O(|U|+|V|)^{4}\right)
\end{aligned}
$$

If the mapping(20) appears the Hopf bifurcation phenomenon, also need the following discriminatory quantity a is not zero[18]

$$
a=-\operatorname{Re}\left(\frac{(1-2 \bar{\lambda}) \bar{\lambda}^{2}}{1-\lambda} \xi_{11} \xi_{20}\right)-\frac{1}{2}\left|\xi_{11}\right|^{2}-\left|\xi_{02}\right|^{2}+\operatorname{Re}\left(\bar{\lambda} \xi_{21}\right) \neq 0
$$

where

$$
\begin{aligned}
& \xi_{20}=\frac{1}{8}\left(F_{U U}-F_{V V}+2 G_{U V}\right)+i\left(G_{U U}-G_{V V}+2 F_{U V}\right) \\
& \xi_{11}=\frac{1}{4}\left(F_{U U}+F_{V V}\right)+i\left(G_{U U}+G_{V V}\right) \\
& \xi_{02}=\frac{1}{8}\left(F_{U U}-F_{V V}-2 G_{U V}\right)+i\left(G_{U U}-G_{V V}+2 F_{U V}\right) \\
& \xi_{21}=\frac{1}{16}\left(F_{U U U}+F_{U V V}+G_{U U V}+G_{V V V}\right)+i\left(G_{U U U}+G_{U V V}-F_{U U V}-F_{V V V}\right) .
\end{aligned}
$$

in which $F=\frac{F_{1}}{b_{12} \alpha_{2}}, G=\frac{G_{1}}{b_{12} \alpha_{2}}, F_{U U}=\left.\frac{\partial^{2} F}{\partial U^{2}}\right|_{U=V=0}, F_{U U U}=\left.\frac{\partial^{3} F}{\partial U^{3}}\right|_{U=V=0}$, and likewise for all other similar terms.
We know from the above derivations: if the conditions (10),(11),(15),(18),(21) is satisfied, the Hopf bifurcation will appear at $\left(N_{3}, P_{3}\right)$.

\section{Conditions for Turing Instability}

The Turing instability requires two conditions: (1) there is a nontrivial homogeneous steady state, and the space homogeneous perturbation is stable, this condition is obtained in Section 3.1. (2) The stationary state is unstable for at least one spatial inhomogeneous perturbation.

A spatial heterogeneity perturbation is introduced to interfere with the stable homogeneous state of $\left(N_{3}, P_{3}\right)$, $\nabla_{d}^{2} N_{(i, j, m)}$ and $\nabla_{d}^{2} P_{(i, j, m)}$ is not always be zero. Before removing the spatial heterogeneous perturbations, the discrete Laplace operator should be determined first.

Firstly, we consider the following equations

$$
\nabla_{d}^{2} X^{i j}+\lambda X^{i j}=0
$$

with periodic boundary conditions

$$
X^{i, 0}=X^{i, n}, X^{i, 1}=X^{i, n+1}, X^{0, j}=X^{n, j}, X^{1, j}=X^{n+1, j}
$$

Apply the methods described in[13], the eigenvalues of the Laplace operator can be formulated as

$$
\lambda_{k l}=4\left(\sin ^{2}\left(\frac{(k-1) \pi}{n}\right)+\sin ^{2}\left(\frac{(l-1) \pi}{n}\right)\right), k, l \in\{1,2,3, \cdots, n\}
$$

Next, we consider the heterogeneous perturbations in the homogeneous space near the $\left(N_{3}, P_{3}\right)$, i.e.,

$$
\begin{aligned}
& N_{(i, j, m)}=N_{3}+\tilde{N}_{(i, j, m)} \\
& P_{(i, j, m)}=P_{3}+\tilde{P}_{(i, j, m)}
\end{aligned}
$$

where $\tilde{N}_{(i, j, m)}$ and $\tilde{P}_{(i, j, m)}$ are the perturbations on the density of prey and predator density in $(i, j)$ site at $m$ th iteration, and noticing that

$$
\begin{aligned}
& \nabla_{d}^{2} N_{(i, j, m)}=\nabla_{d}^{2} \tilde{N}_{(i, j, m)} \\
& \nabla_{d}^{2} P_{(i, j, m)}=\nabla_{d}^{2} \tilde{P}_{(i, j, m)}
\end{aligned}
$$

Substituting Eqs.(25) into Eqs.(4) can obtain 


$$
\begin{aligned}
\tilde{N}_{(i, j, m+1)} & =b_{11}\left(\tilde{N}_{(i, j, m)}+\frac{\tau}{h^{2}} d_{1} \nabla_{d}^{2} \tilde{N}_{(i, j, m)}\right)+b_{12}\left(\tilde{P}_{(i, j, m)}+\frac{\tau}{h^{2}} d_{2} \nabla_{d}^{2} \tilde{P}_{(i, j, m)}\right) \\
& +O\left(\left(\left|\tilde{N}_{(i, j, m)}\right|+\left|\tilde{P}_{(i, j, m)}\right|\right)^{2}\right) \\
\tilde{P}_{(i, j, m+1)} & =b_{21}\left(\tilde{N}_{(i, j, m)}+\frac{\tau}{h^{2}} d_{1} \nabla_{d}^{2} \tilde{N}_{(i, j, m)}\right)+b_{22}\left(\tilde{P}_{(i, j, m)}+\frac{\tau}{h^{2}} d_{2} \nabla_{d}^{2} \tilde{P}_{(i, j, m)}\right) \\
& +O\left(\left(\left|\tilde{N}_{(i, j, m)}\right|+\left|\tilde{P}_{(i, j, m)}\right|\right)^{2}\right)
\end{aligned}
$$

when the perturbation is very small, the $O\left(\left(\left|\tilde{N}_{(i, j, m)}\right|+\left|\tilde{P}_{(i, j, m)}\right|\right)^{2}\right)$ can be neglected. By using the eigenvalue $\lambda_{k l}$, the corresponding characteristic function $X_{k l}^{i j}$, multiplied by the above formula, can be obtained

$$
\begin{aligned}
X_{k l}^{i j} \tilde{N}_{(i, j, m)}= & b_{11} X_{k l}^{i j} \tilde{N}_{(i, j, m)}+b_{12} X_{k l}^{i j} \tilde{P}_{(i, j, m)}+\frac{\tau}{h^{2}} b_{11} d_{1} X_{k l}^{i j} \nabla_{d}^{2} \tilde{N}_{(i, j, m)} \\
& +\frac{\tau}{h^{2}} b_{12} d_{2} X_{k l}^{i j} \nabla_{d}^{2} \tilde{P}_{(i, j, m)} \\
X_{k l}^{i j} \tilde{P}_{(i, j, m)}= & b_{21} X_{k l}^{i j} \tilde{N}_{(i, j, m)}+b_{22} X_{k l}^{i j} \tilde{P}_{(i, j, m)}+\frac{\tau}{h^{2}} b_{21} d_{1} X_{k l}^{i j} \nabla_{d}^{2} \tilde{N}_{(i, j, m)} \\
& +\frac{\tau}{h^{2}} b_{22} d_{2} X_{k l}^{i j} \nabla_{d}^{2} \tilde{P}_{(i, j, m)}
\end{aligned}
$$

Summing Eqs.(28) for all of $i$ and $j$ obtains

$$
\begin{aligned}
\sum_{i, j=1}^{n} X_{k l}^{i j} \tilde{N}_{(i, j, m+1)} & =b_{11} \sum_{i, j=1}^{n} X_{k l}^{i j} \tilde{N}_{(i, j, m)}+b_{12} \sum_{i, j=1}^{n} X_{k l}^{i j} \tilde{P}_{(i, j, m)} \\
& +\frac{\tau}{h^{2}} b_{11} d_{1} \sum_{i, j=1}^{n} X_{k l}^{i j} \nabla_{d}^{2} \tilde{N}_{(i, j, m)}+\frac{\tau}{h^{2}} b_{12} d_{2} \sum_{i, j=1}^{n} X_{k l}^{i j} \nabla_{d}^{2} \tilde{P}_{(i, j, m)} \\
\sum_{i, j=1}^{n} X_{k l}^{i j} \tilde{P}_{(i, j, m+1)} & =b_{21} \sum_{i, j=1}^{n} X_{k l}^{i j} \tilde{N}_{(i, j, m)}+b_{22} \sum_{i, j=1}^{n} X_{k l}^{i j} \tilde{P}_{(i, j, m)} \\
& +\frac{\tau}{h^{2}} b_{21} d_{1} \sum_{i, j=1}^{n} X_{k l}^{i j} \nabla_{d}^{2} \tilde{N}_{(i, j, m)}+\frac{\tau}{h^{2}} b_{22} d_{2} \sum_{i, j=1}^{n} X_{k l}^{i j} \nabla_{d}^{2} \tilde{P}_{(i, j, m)}
\end{aligned}
$$

Let $\bar{N}_{m}=\sum_{i, j=1}^{n} X_{k l}^{i j} \tilde{N}_{(i, j, m)}$ and $\bar{P}_{m}=\sum_{i, j=1}^{n} X_{k l}^{i j} \tilde{P}_{(i, j, m)}$, Eqs.(34) can be transformed into the following form

$$
\begin{aligned}
& \bar{N}_{m+1}=b_{11}\left(1-\frac{\tau}{h^{2}} d_{1} \lambda_{k l}\right) \bar{N}_{m}+b_{12}\left(1-\frac{\tau}{h^{2}} d_{2} \lambda_{k l}\right) \bar{P}_{m} \\
& \bar{P}_{m+1}=b_{21}\left(1-\frac{\tau}{h^{2}} d_{1} \lambda_{k l}\right) \bar{N}_{m}+b_{22}\left(1-\frac{\tau}{h^{2}} d_{2} \lambda_{k l}\right) \bar{P}_{m}
\end{aligned}
$$

If the Eqs(30) converges, the discrete predator-prey system will return to the homogeneous state again; if divergent, the homogeneous state will be destroyed and the Turing patterns will be formed.

Calculating the two eigenvalues of the Jacobi matrix of (30)

$$
\begin{aligned}
& \lambda_{ \pm}(k, l)=\frac{1}{2}\left(b_{11}+b_{22}-\frac{\tau}{h^{2}}\left(b_{11} d_{1}+b_{22} d_{2}\right) \lambda_{k l}\right) \\
& \quad \pm \frac{1}{2} \sqrt{\left(b_{11}-b_{22}-\frac{\tau}{h^{2}}\left(b_{11} d_{1}-b_{22} d_{2}\right) \lambda_{k l}\right)^{2}+4 b_{12} b_{21}\left(1-\frac{\tau}{h^{2}} d_{1} \lambda_{k l}\right)\left(1-\frac{\tau}{h^{2}} d_{2} \lambda_{k l}\right)}
\end{aligned}
$$

Whether $\left|\lambda_{+}(k, l)\right|>1$ or $\left|\lambda_{-}(k, l)\right|>1$ exists, there will be at least one group of $k$ and $l$ satisfy $\lambda_{k l} \neq 0$, and then (30) will converge. The divergence Eqs.(30) suggests the occurrence of Turing instability. Next, we defines

$$
Z(k, l)=\max \left(\left|\lambda_{+}(k, l)\right|,\left|\lambda_{-}(k, l)\right|\right)
$$

and when

$$
Z_{m}=\max _{k=1}^{n} \max _{l=1}^{n} Z(k, l),(k, l) \neq(1,1)
$$

Turing instability occurs. Therefore, condition(32) is the criterion for judging Turing instability.

\section{NUMERICAL SIMULATIONS}

In order to use numerical methods to illustrate the theoretical results deduced in Section 3, we select a set of parameters to verify the parameter conditions of Hopf bifurcation and Turing instability. Parameters are selected as follows

$$
\begin{gathered}
\varepsilon=0.2, a=0.1, b=0.2, \beta=0.2, \gamma=0.1, K=2, \\
\delta=0.25, \tau=0.01, h=0.5, d_{1}=1.5, d_{2}=1.5
\end{gathered}
$$

The positive equilibrium point is $\left(N_{3}, P_{3}\right)=(1.3713,3.9516)$. Substituting parameters into in the parameter conditions for the existence of Hopf bifurcation and Turing instability in Section 3 , we can find that all conditions are satisfied.

We take $\mathrm{K}$ and $\mathrm{d}_{1}$ of the set of parameters(33) as variables, we can obtain a bifurcation diagram, as shown in Figure II. It shows four cases of the discrete predator-prey model, which separated by the curves of Hopf bifurcation and Turing instability. Region I is the case of stable state without Hopf bifurcation and Turing instability; region II is the case of pure Hopf bifurcation; region III is the case of pure Turing instability; and region IV is the case of the presence of both Hopf bifurcation and Turing instability. According to the theories of Turing instability, we can know the region III and IV can give rise to spatial patterns.
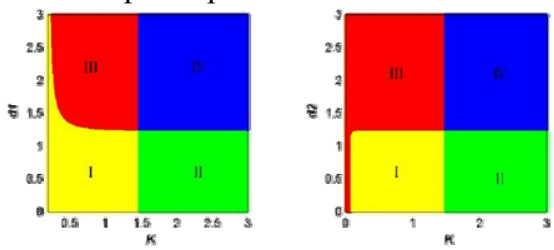

FIGURE II. REGION DIAGRAMS SHOWN IN K -D1 AND K - D2. THE REGIONS ARE SEPARATED BY THE CURVES OF HOPFBIFURCATION AND TURING INSTABILITY. $\mathrm{T}=0.01, \mathrm{H}=$ 0.5 , THE OTHER PARAMETER VALUES IN THIS GRAPH IS SAME WITH THAT IN(33)

Next, for convenience of observation, we only show the change of the prey patterns, the change of predator patterns is similar. Now, we fix the values of the following parameters as 
$\varepsilon=0.2, a=0.1, b=0.2, \beta=0.2, \gamma=0.1, \delta=0.25$
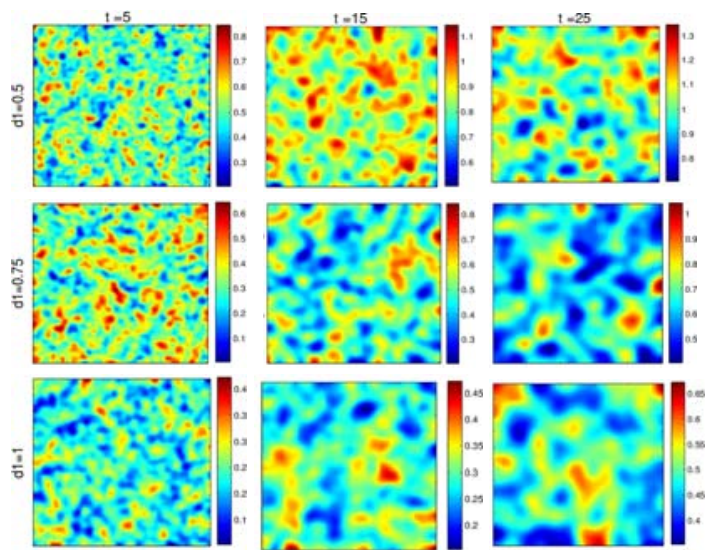

FIGURE III. THE CHANGES OF SPATIAL PATTERNS FOR PREY WITH DIFFERENT VALUES OF D1.(1) D1 $=0.5$, (2) D1 $=0.75$, (3) D1 $=1$. THE OTHER PARAMETER VALUES IN EACH GRAPH ARE THE SAME WITH THAT IN (34).

and shift the values of the other parameters $K, \tau, h$ and $d_{l}$ to observe the dynamical variations in the time- and spacediscrete predator-prey model.
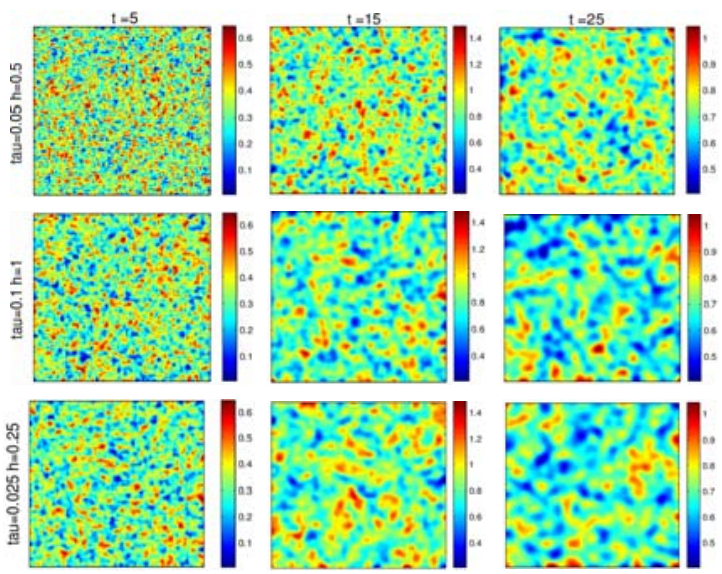

FIGURE IV. SPATIAL PATTERNS FOR PREY WITH DIffERENT VALUES H AND T . (1) $\mathrm{T}=0.1, \mathrm{H}=1,(2) \mathrm{T}=0.5, \mathrm{H}=0.05,(3) \mathrm{T}=$ $0.025, \mathrm{H}=0.25$. THE OTHER PARAMETER VALUES ARE $\mathrm{E}=0.2$, $\mathrm{A}=0.1, \mathrm{~B}=0.2, \mathrm{~K}=2, \mathrm{~B}=0.2, \Gamma=0.1, \Delta=0.25, \mathrm{D} 1=1.5$

Figure III. shows the change the value of $d_{1}$ may fragment the patterns of prey. We can find that the variation of the value $d_{l}$ can change the diffusion capability of prey and influence the diffusion process. When reducing the value of $d_{l}$, the patterns become spatially symmetrical from irregular shapes. The patterns is becoming smaller and more stable when fix the value of $d_{l}$. Figure IV. shows the variations of prey spatial patterns with three groups $\tau$ and $h$. We find that changing the value of $\tau$ and $h$ can change the shape of the patterns.

\section{CONCLUSIONS}

Hopf bifurcation and Turing instability of a space- and time- discrete model with Holling type II function response are investigated in this paper. It has been proven that the discrete system exists Hopf bifurcation and Turing instability, and the parameter conditions of Hopf bifurcation and Turing instability are obtained. Applying the bifurcation theory of discrete system, we obtain the discrete system will undergo three instability mechanisms under the certain parameter conditions at $(\bar{N}, \bar{P})$, including Hopf instability, Turing instability and Hopf-Turing instability.

\section{ACKNOWLEDGMENTS}

This work is jointly supported by 2015 National traditional Medicine Clinical Research Base Business Construction Special Topics (JDZX2015299) and the Fundamental Research Funds for the Central Universities FRF-BR-16-019A.

\section{REFERENCES}

[1] Thompson D J. Towards a predator-prey model incorporating age structure: the effects of predator and prey size on the predation of Daphnia magna by Ischnura elegans[J]. The Journal of Animal Ecology, 1975: 907-916.

[2] Lee S, Ahn I. Diffusive predator-prey models with stage structure on prey and Beddington-DeAngelis functional responses[J]. Communications on Pure \& Applied Analysis, 2017, 16(2):427-441.

[3] Chang L, Sun G Q, Wang Z, et al. Rich dynamics in a spatial predatorCprey model with delay[J]. Applied Mathematics and Computation, 2015, 256: 540-550.

[4] Peng Y, Zhang T. Turing instability and pattern induced by crossdiffusion in a predator-prey system with Allee effect[M]. Elsevier Science Inc. 2016.

[5] Ren J, Yu L, Siegmund S. Bifurcations and chaos in a discrete predatorCprey model with CrowleyCMartin functional response[J]. Nonlinear Dynamics, 2017(352):1-23

[6] V. Castets, E. Dulos, J. Boissonade, P. De Kepper, Experimental evidence for a sustained Turing-type nonequilibrium chemical pattern, Phys. Rev. Lett. 64(1990) 2953C2956.

[7] Q. Ouyang, H,L. Swinney, Transition to chemical turbulence, Chaos 1 (1991)411C420

[8] Domokos G, Scheuring I. Discrete and continuous state population models in a noisy world[J]. Journal of Theoretical Biology, 2004, 227(4):535-45

[9] Saratchandran PP , Ajithprasad KC , Harikrishnan KP . Numerical exploration of the parameter plane in a discrete predator-prey model. Ecol Complexity 2015;21:112C19.

[10] Cavani M, Farkas M. Bifurcations in a predator-prey model with memory and diffusion II: Turing bifurcation[J]. Acta Mathematica Hungarica, 1994, 63(4):375-393.

[11] Nayfeh AH , Balachandran B . Applied nonlinear dynamics: analytical, computational, and experimental methods. Wiley Interscience; 1995 .

[12] Guckenheimer J , Holmes P . Nonlinear oscillations, dynamical systems and bifurcations of vector fields. NY: Springer-Verlag; 1983 .

[13] Bai L , Zhang G . Nontrivial solutions for a nonlinear discrete elliptic equation with periodic boundary conditions. Appl Math Comput 2009;210:321C33. 